\title{
Indicadores de boas práticas na assistência neonatal ao parto em maternidade terciária em Fortaleza
}

\section{Indicators of good practices in neonatal assistance during birth in tertiary maternity in Fortaleza}

Mara Larissa Alves Marques Patrício ${ }^{1,2}$. Liliana Soares Nogueira Paes ${ }^{1}$. Eveline Campos Monteiro de Castro ${ }^{1}$. Gerly Anne Nóbrega Barreto1. Marcos Paulo Fernandes Patrício².

1 Maternidade Escola Assis Chateaubriand (MEAC/UFC/EBSERH), Fortaleza, Ceará, Brasil. 2 Hospital Universitário Walter Cantídio (HUWC/UFC/EBSERH), Fortaleza, Ceará, Brasil.

\section{RESUMO}

Objetivos: Avaliar a aplicação das boas práticas na assistência neonatal em maternidade terciária. Metodologia: Estudo observacional transversal retrospectivo de base hospitalar, com análise dos neonatos elegíveis para boas práticas nascidos entre 01/01/2017 e 30/06/2017. Resultados: avaliaram-se 2.481 nascimentos e 1.930 pacientes foram elegíveis. Desses, $46.9 \%$ nasceram de partos vaginais e $53.1 \%$ de cesáreas; 1038 eram do sexo masculino $(52.5 \%)$ e 88 neonatos, gemelares. Encontrou-se peso médio ao nascer de $3134 \pm 539$ gramas e idade gestacional média de $38.54 \pm 1.8$ semanas. Realizou-se clampeamento do cordão entre 1 a 3 minutos em 79,4\%, contato pele a pele em $66,7 \%$ e amamentação na primeira hora de vida em $44,3 \%$ dos nativivos elegíveis. Conclusão: a adesão quanto ao clampeamento oportuno do cordão umbilical foi alta, no entanto, observou-se menor adesão ao contato pele a pele e à amamentação na $1^{\text {a }}$ hora de vida. As principais causas de não aplicabilidade foram prematuridade e comprometimento da vitalidade do recém-nascido. É importante disponibilizar mais profissionais, avaliar a indicação de prematuros tardios e sensibilizar a equipe e as mães da relevância das boas práticas para melhor adesão.

Palavras-chave: Aleitamento materno. Recém-nascido. Recém-nascido prematuro. Mortalidade infantil.

\section{ABSTRACT}

Objectives: To evaluate the application of good practices in neonatal care in tertiary maternity. Methodology: Cross-sectional observational, hospital-based observational study with analysis of neonates eligible for good practices born between 01/01/2017 and 06/30/2017. Results: 2.481 births were evaluated, and 1.930 patients were eligible. Of these, $46.9 \%$ were born of vaginal deliveries and $53.1 \%$ were cesarean; 1.038 were male (52.5\%) and 88 were neonates, twins. The mean birth weight was $3.134 \pm$ 539 grams and mean gestational age was $38.54 \pm 1.8$ weeks. Cord clamping was performed between 1 to 3 minutes in $79.4 \%$, skinto-skin contact in $66.7 \%$, and breastfeeding in the first hour of life in $44.3 \%$ of eligible children. Conclusion: timely clamping of the umbilical cord was high; however, adherence to skin-to-skin contact and breastfeeding in the first hour of life were observed. The most important reasons of non-applicability were prematurity and impairment of vitality. It is important to make more professionals available to evaluate the indication of late preterm infants and to make the team and the mothers aware of the relevance of good practices for better adherence.

Keywords: Breastfeeding. Newborn. Premature newborn. Child mortality.

Autor correspondente: Mara Larissa Alves Marques Patrício, Rua Bruno Porto, 555, Parque Iracema, Fortaleza, Ceará. CEP: 60824-010. Telefone: +55 85 99403-7412. E-mail: maralarissam@gmail.com

Conflito de interesses: Não há qualquer conflito de interesses por parte de qualquer um dos autores.

Recebido em: 11 Fev 2019; Revisado em: 29 Jul 2020; Aceito em: 02 Jul 2021. 


\section{INTRODUÇÃO}

A mortalidade neonatal é o principal componente da mortalidade infantil no Brasil desde a década de 1990 e, embora esteja em queda, persiste em níveis elevados. ${ }^{1}$ Em 2018, dos 20.272 óbitos infantis, 16.026 ocorreram na primeira semana de vida (6,3 por mil nascidos vivos), principalmente nas primeiras 24 horas, ${ }^{2,3}$ indicando estreita relação com a atenção ao parto e nascimento. ${ }^{1}$ As principais causas de óbitos nessa faixa segundo a literatura são: prematuridade, malformação congênita, asfixia intra-parto, infecções perinatais e fatores maternos, com uma proporção considerável de mortes preveníveis por ação dos serviços de saúde. ${ }^{4}$

O atendimento ao recém-nascido a termo saudável na sala de parto têm sofrido modificações nos últimos anos e um dos objetivos é diminuir o excesso de intervenções logo após o nascimento. Portanto, tem sido recomendado que práticas dirigidas a outras questões não ligadas exclusivamente à sobrevivência precisam ser incorporadas aos cuidados de rotina do recém-nascido. ${ }^{5}$

As boas práticas na assistência neonatal ao parto incluem: clampeamento oportuno do cordão umbilical, entre um e três minutos após a extração do concepto, ${ }^{6}$ contato pele a pele imediato mãe-bebê, quando o recém-nascido é colocado envolto em campos aquecidos em contato direto com a mãe, ao nascer ou logo após ${ }^{7}$ início precoce do aleitamento materno até uma hora após o nascimento. Entretanto, a utilização dessas práticas na assistência ao parto e ao recém-nascido sofre grande variação entre unidades de saúde no mundo, coexistindo ainda com desigualdades geográficas e sociais, diferenças na expertise médica e características demográficas dos pacientes. ${ }^{5}$

O clampeamento oportuno do cordão umbilical é recomendado como estratégia fácil e de baixo custo para melhorar os níveis de ferritina ao nascimento e prevenir a anemia na infância. ${ }^{8}$

O contato pele a pele precoce entre mãe e filho e a amamentação na primeira hora de vida do bebê representam importantes estratégias para a promoção do aleitamento materno (AM). Essas orientações são preconizadas pela Organização Mundial da Saúde (OMS) e pelo Fundo das Nações Unidas para a Infância (UNICEF, United Nations Children's Found). As estratégias visam a promoção, proteção e apoio ao AM e se baseia na capacidade de interação dos recém-nascidos com suas mães, logo após o nascimento, assim, deve ser incentivada ainda na sala de parto, desde que mãe e filho estejam bem. ${ }^{9} \mathrm{O}$ contato corporal mãe/bebê contribui para a termorregulação do neonato, a manutenção do equilíbrio acidobásico, o ajuste da respiração e do choro, e promove o comportamento cuidador materno. $^{10}$

O incentivo para o início da amamentação neste período de maior sensibilidade materna é uma prática que pode reduzir infecção neonatal e mortalidade neonatal em $22 \%$. $^{11,12}$

Vários mecanismos podem explicar o efeito protetor da amamentação na primeira hora de vida, como: colonização intestinal do recém-nascido por bactérias saprófitas encontradas no leite materno, ${ }^{13}$ propriedades do leite materno de reduzir a colonização intestinal por bactérias gram-negativas $;{ }^{14}$ capacidade adaptativa materna em produzir fatores imunológicos bioativos adequados para o recém-nascido que são excretados no colostro de acordo com a idade gestacional; ${ }^{15}$ e presença de imunoglobulina A, que apresenta maior concentração no colostro quando comparado com o leite maduro. ${ }^{16}$

Em maio de 2014, o Ministério da Saúde brasileiro institui diretrizes para a organização da atenção integral e humanizada ao recém-nascido (RN) no Sistema Único de Saúde (SUS), com as recomendações de assegurar o contato pele a pele imediato e contínuo; proceder ao clampeamento do cordão umbilical, após 1 a 3 minutos, exceto em casos de mães isoimunizadas ou HIV e HTLV positivas; estimular o aleitamento materno na primeira hora de vida, exceto em casos de mães HIV ou HTLV positivas e postergar os procedimentos de rotina do recém-nascido nessa primeira hora de vida. ${ }^{17}$

A implementação das boas práticas no trabalho de parto e parto é uma ação potente para prevenção dos óbitos neonatais evitáveis. Assim, o objetivo deste estudo é avaliar os indicadores de boas práticas na assistência ao recém-nascido durante o parto em maternidade de referência na cidade de Fortaleza/CE - Brasil.

\section{METODOLOGIA}

Trata-se de um estudo observacional transversal retrospectivo, de base hospitalar. O estudo foi submetido ao Comitê de Ética e Pesquisa da referida maternidade, com aprovação de acordo com o parecer 2.423.568.

A Maternidade Escola Assis Chateaubriand (MEAC) é referência em assistência ao parto pelo Ministério da Saúde. São monitorados continuamente indicadores de assistência à mulher, ao parto e ao recém-nascido. Esses são obtidos de formulários preenchidos nos serviços onde mãe e/ou filho são atendidos e posteriormente digitados em banco de dados. Constam nos relatórios anuais de gestão.

$\mathrm{Na}$ coleta de dados utilizou-se a ficha de monitoramento/ avaliação da assistência ao parto e nascimento do centro obstétrico da MEAC (onde são informados dados demográficos, intervenções, aplicabilidade das boas práticas e destino do recém-nascido) e o livro de registros de partos da neonatologia. Os dados foram coletados pela própria pesquisadora.

O estudo foi realizado no período de 01/01/2017 a 30/06/2017. Houve 2.516 partos no período do estudo, mas foram encaminhadas 2.481 fichas de monitoramento para análise. Não se sabe o perfil das fichas não enviadas. Foram considerados critérios de inclusão maternos: estar em boas condições clínicas para receber o filho e não ter o diagnóstico de retrovirose no pré-natal ou em teste rápido anteparto; e neonatais: pacientes nascidos no centro obstétrico da 
maternidade, com idade gestacional (IG) maior ou igual a 34 semanas e peso de nascimento (PN) maior que $1500 \mathrm{~g}$, boa vitalidade e sem uso de oxigênio suplementar por capacete ou suporte ventilatório.

Excluíram-se os recém-nascidos com idade gestacional inferior a 34 semanas ou peso de nascimento menor que $1500 \mathrm{~g}$, malformações maiores e/ou síndromes genéticas, comprometimento de vitalidade (por desconforto respiratório com necessidade de oxigênio suplementar em capacete ou suporte ventilatório, admissão em unidade de terapia intensiva neonatal (UTIN) ou unidade de cuidados intermediários convencionais (UCINCO); escore de Apgar inferior a 7 no quinto minuto de vida), filhos de mães admitidas em UTI materna ou com gravidade que impossibilitasse fazer o contato pele a pele e filhos de mães com sorologia positiva para vírus da imunodeficiência humana (HIV), bem como os neonatos nascidos fora do centro obstétrico da maternidade.

Os dados foram compilados por meio de questionário estruturado (Quadro 1), colocados em planilha do Microsoft Excel $^{\circledR}$ e então realizada análise, com criação de tabelas e realização do teste Qui-quadrado, Teste T de Student, Kruskall-Wallis, além do teste Post-Hoc para comparações múltiplas para avaliação dos dados. O programa utilizado para a análise dos dados foi o SPSS 22.

Quadro 1. Questionário para coleta de dados.

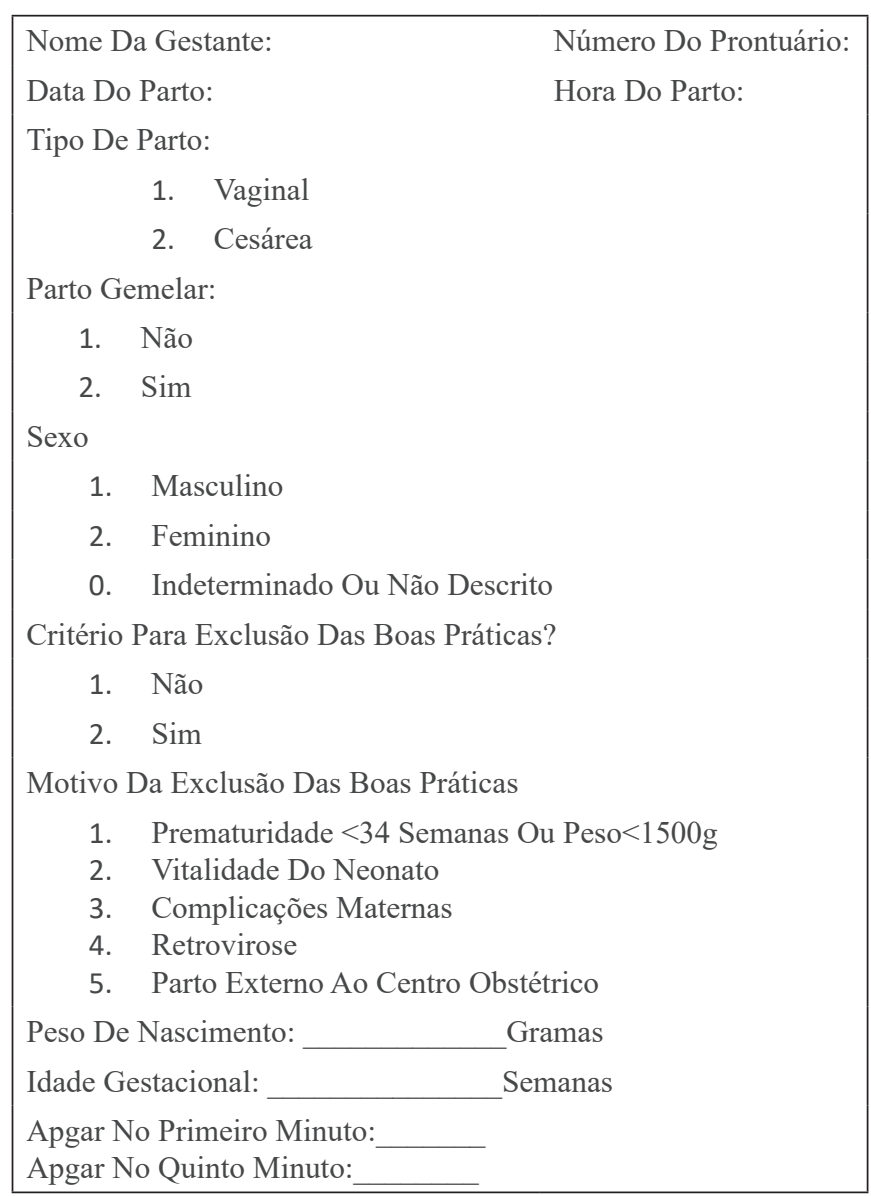

Continua.
Conclusão.

Quadro 1. Questionário para coleta de dados.

Realizou Contato pele a pele?
1. Não
2. Sim
0. Não descrito

Porque Não Realizou As Boas Práticas?
1. Prematuridade $<34$ Semanas Ou Peso $<1500 \mathrm{~g}$
2. Vitalidade Do Neonato
3. Complicações Maternas
4. Retrovirose
5. Parto Externo Ao Centro Obstétrico
6. Ausência De Profissional Disponível

O Neonato Foi Amamentado Na Primeira Hora De Vida?
1. Não
2. Sim
$0 . \quad$ Não Informado

Em Quanto Tempo Foi Realizado O Clampeamento Do Cordão?
1. $<1$ Minuto
2. Entre 1-3 Minutos
3. $>3$ Minutos
0 . Não informado

O Neonato Necessita De Oxigênio Suplementar?
1. Não
2. Sim
0. Não Informado

O Neonato Foi Reanimado Na Sala De Parto?
1. Não
2. Sim
0. Não Informado

O Neonato Recebeu Reanimação Com Vpp/ Iot?
1. Não
2. Sim
0. Não Informado

O Neonato Recebeu Reanimação Avançada Com Massagem Cardíaca E Drogas?
1. Não
2. $\mathrm{Sim}$
0. Não Informado

Quem Assistiu Ao Paciente Ao Nascer?
1. Neonatologista
2. Médico Residente Em Neonatologia Ou Pediatria
3. Enfermeiro Neonatologista
0 . Não descrito

Qual A Unidade De Destino Do Recém-Nascido?
1. Alojamento Conjunto
2. UCINCo
3. UTI Neonatal
0. Não descrito 


\section{RESULTADOS}

Nasceram no período do estudo 2.516 neonatos. Foram enviadas 2.481 fichas de monitoramento/avaliação da assistência ao parto e nascimento. Não se obteve acesso a 35 fichas $(1,4 \%)$ por não terem sido encaminhadas. Entre os nascidos vivos, 551 não foram elegíveis para boas práticas ao RN (Figura 1).

Entre os pacientes em condições de se realizar as boas práticas na primeira hora de vida, a maioria foi descrita como sexo masculino, $1.014(52,5 \%), 88(4,8 \%)$ foram nativivos de gestações gemelares e a via de parto predominante foi a cesárea, 1.025 casos. Os dados demográficos da população elegível estão na Tabela 1.

Figura 1. Algoritmo de seleção dos pacientes.

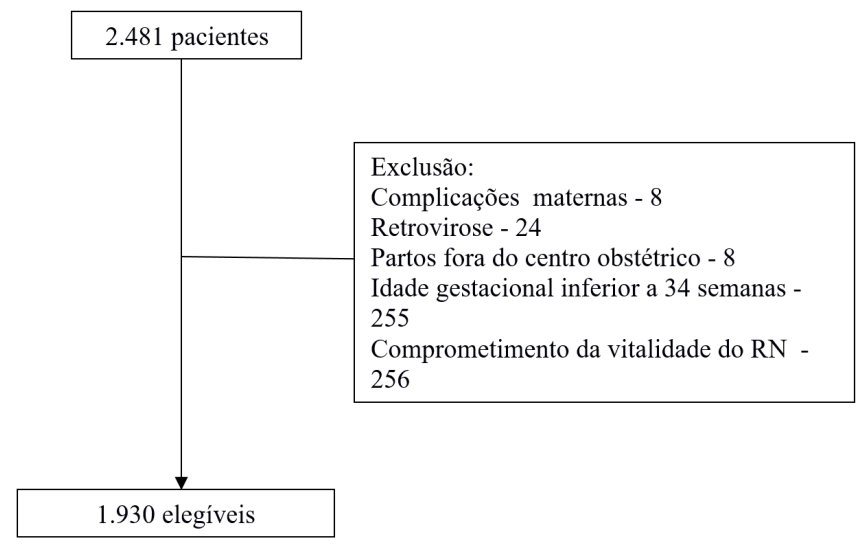

Tabela 1. Características demográficas da população elegível.

\begin{tabular}{lc}
\hline & $\begin{array}{c}\text { Nativivos Elegíveis } \\
\mathrm{N}=1930\end{array}$ \\
\hline Tipo de Parto & $905(46,9)$ \\
Vaginal & $1025(53,1)$ \\
Cesárea & \\
Sexo & $1014(52,6)$ \\
Masculino & $914(47,3)$ \\
Feminino & $2(0,1)$ \\
Indeterminado/NãoDescrito & \\
Gemelaridade & $88(4,6)$ \\
$\quad$ Sim & $1.841(95,4)$ \\
$\quad$ Não & $38,54 \pm 1,78)$ \\
Idade Gestacional (semanas) & $3.134 \pm 539$ \\
Peso (gramas) & $9(8-9)$ \\
Apgar $1^{\circ}$ minuto & $9(9-9)$ \\
Apgar 5 ${ }^{\circ}$ minuto &
\end{tabular}

Dados expressos em números absolutos e porcentagens (\%) ou média \pm desvio padrão (DP) ou mediana (P25-75).
Foi necessária ventilação com pressão positiva em 41 pacientes $(2,1 \%)$, não sendo realizada reanimação avançada com massagem cardíaca ou drogas em nenhum dos casos. Utilizou-se oxigênio suplementar em algum momento em 137 (7,0\%) pacientes. A maioria dos pacientes foi encaminhado ao alojamento conjunto, 1.657 neonatos $(85,8 \%)$ (Tabela 2$)$.

$\mathrm{Na}$ análise de indicadores de boas práticas ao nascer, aquele com maior aplicabilidade entre a população estudada foi o clampeamento oportuno do cordão umbilical, realizado em $1.372(79,4 \%)$ casos. O indicador de menor aplicabilidade foi a amamentação na primeira hora de vida, realizada em apenas $760(39,3 \%)$ dos 1.930 elegíveis.

Tabela 2. Intervenções realizadas nos pacientes elegíveis e unidades de admissão hospitalar.

\begin{tabular}{lc}
\hline \multicolumn{2}{c}{ Nativivos elegíveis } \\
\hline Uso de Oxigênio Suplementar \\
Não informado & $214(11,0)$ \\
Não & $1.579(81,8)$ \\
Sim & $137(7,0)$ \\
Reanimação na Sala de Parto & $131(6,7)$ \\
Não informado & $1.758(91)$ \\
Não & $41(2,1)$ \\
Sim & $2(4,9)$ \\
Reanimação com VPP $/$ IOT $^{+}$ & $0(0,0)$ \\
Não informado & $39(95,1)$ \\
Não & \\
Sim & $30(73,2)$ \\
Reanimação Avançada & $11(26,8)$ \\
Não informado & $0(0,0)$ \\
Não & \\
Sim & $1657(85,8)$ \\
Unidade de Admissão & $204(10,5)$ \\
Alojamento Conjunto & $0(0,0)$ \\
UCINCO & $69(35)$ \\
UTIN** & \\
Não informado & \\
\hline
\end{tabular}

Dados expressos em números absolutos e porcentagens (\%); *VPP: Ventilação com Pressão Positiva, +IOT: Intubação Oro Traqueal, §UCINCO: Unidade de Cuidados Intermediários, ** UTIN: Unidade de Terapia Intensiva Neonatal.

O clampeamento oportuno do cordão umbilical esteve presente em $675(72 \%)$ cesáreas e $697(88,1 \%)$ partos vaginais. Em $51(60,7 \%)$ partos gemelares houve clampeamento do cordão umbilical em menos de um minuto de vida. O clampeamento foi precoce (menos de um minuto) na maioria dos pacientes com necessidade de reanimação (37-86\%) e em cerca de metade daqueles que fizeram uso de oxigênio (64 pacientes-51,6\%) (Tabela 3). 
O contato pele a pele foi realizado em $84,8 \%$ dos partos vaginais, no entanto, em apenas $503(51,1 \%)$ das cesáreas. A maioria dos gemelares, 65 (77,4\%), não realizou contato pele a pele na primeira hora de vida. Mesmo com necessidade de reanimação inicial, $11(5,6 \%)$ recém-nascidos tiveram condições de realizar esse indicador ainda na primeira hora de vida, bem como 39 (30,5\%) que em algum momento na primeira hora utilizaram oxigênio (Tabela 4).

A amamentação na primeira hora de vida foi realizada em 549 $(68,9 \%)$ partos vaginais e em $211(23,0 \%)$ partos abdominais.
Apenas seis $(7,1 \%)$ gemelares foram amamentados na primeira hora de vida. Receberam reanimação quatro $(9,5 \%)$ dos bebês que foram amamentados, e nove $(7,1 \%)$ receberam oxigênio (Tabela 5).

Quando se considerou a aplicabilidade dos três indicadores, $61,9 \%$ dos nascidos de parto vaginal conseguiram realizar as três práticas, em contraponto a $19,6 \%$ dos nascidos de cesárea. Apenas $5(5,9 \%)$ dos gemelares realizaram os três indicadores, bem como dois dos elegíveis que foram reanimados e cinco dos que receberam oxigênio.

Tabela 3. Clampeamento oportuno do cordão umbilical.

\begin{tabular}{|c|c|c|c|c|}
\hline \multicolumn{5}{|c|}{ Clampeamento oportuno do cordão umbilical } \\
\hline & $<1 \min$ & $1-3 \mathrm{~min}$ & $>3 \mathrm{~min}$ & $p$ \\
\hline Tipo de Parto & & & & $<0,01$ \\
\hline Vaginal & $82(10,4)$ & $697(88,1)$ & $12(1,5)$ & \\
\hline Cesárea & $261(27,9)$ & $675(72,0)$ & $1(0,1)$ & \\
\hline Gemelaridade & & & & $<0,01$ \\
\hline Sim & $51(60,7)$ & $32(38,1)$ & $1(1,2)$ & \\
\hline Não & $292(17,8)$ & $1339(81,5)$ & $12(0,7)$ & \\
\hline I.G.* (semanas) & $37,9 \pm 2,00$ & $38,7 \pm 1,7$ & $39,01 \pm 1,8$ & $<0,01$ \\
\hline P.N. ${ }^{+}$(gramas) & $2.962 \pm 605$ & $3.178 \pm 513$ & $3.277 \pm 444$ & $<0,01$ \\
\hline Apgar $1^{\circ}$ minuto & $8(7-9)$ & $9(8-9)$ & $8(8-9)$ & $<0,01$ \\
\hline Apgar $5^{\circ}$ minuto & $9(9-9)$ & $9(9-9)$ & $9(9-9)$ & $<0,01$ \\
\hline Reanimacão & & & & $<0,01$ \\
\hline Sim & $37(86,0)$ & $6(14,0)$ & $0(0,0)$ & \\
\hline Não & $301(18,3)$ & $1333(80,9)$ & $13(0,8)$ & \\
\hline Não Informado & $5(13,2)$ & $33(86,8)$ & $0(0,0)$ & \\
\hline Uso De Oxigenio & & & & $<0,01$ \\
\hline Sim & $60(48,4)$ & $64(51,6)$ & $0(0,0)$ & \\
\hline Não & $711(48,0)$ & $769(52,0)$ & & \\
\hline Não informado & $30(28,3)$ & $74(69,8)$ & $2(1,9)$ & \\
\hline
\end{tabular}

Dados expressos em números absolutos e porcentagens (\%) ou média \pm desvio padrão (DP) ou mediana (P25-75).

Tabela 4. Contato pele a pele na população estudada.

\begin{tabular}{lccc}
\hline & \multicolumn{2}{c}{ Contato pele a pele } & \\
\hline & Sim & Não & $<0,01$ \\
Tipo de Parto & & $129(15,2)$ & \\
Vaginal & $719(84,8)$ & $482(48,9)$ & $<0,01$ \\
Cesárea & $503(51,1)$ & & \\
Gemelaridade & & $65(77,4)$ & $<0,01$ \\
Sim & $19(22,6)$ & $1.202(68,8)$ & $<0,01$ \\
Não & $546(31,2)$ & $37,93 \pm 1,97$ & $<0,01$ \\
I.G.* (semanas) & $38,8 \pm 1,54$ & $2.994 \pm 616$ & $<0,01$ \\
P.N. ${ }^{+}$(gramas) & $3.208 \pm 478)$ & $8(8-9)$ & $9(9-9)$ \\
Apgar 1 ${ }^{\circ}$ minuto & $9(8-9)$ & $9(9-9)$ & \\
Apgar 5 ${ }^{\circ}$ minuto & &
\end{tabular}

Continua. 
Conclusão.

Tabela 4. Contato pele a pele na população estudada.

\begin{tabular}{lccc}
\hline \multicolumn{2}{c}{ Contato pele a pele } & $<0,01$ \\
\hline Reanimação & $11(5,6)$ & $32(94,4)$ & \\
Sim & $1.177(67,6)$ & $564(32,4)$ & \\
Não & $34(69,4)$ & $15(30,6)$ & $<0,01$ \\
Não Informado & & & \\
Uso De Oxigenio & $39(30,5)$ & $89(69,5)$ & \\
Sim & $1.095(69,8)$ & $474(30,2)$ & \\
Não & $88(64,7)$ & $48(35,3)$ & \\
Não informado & &
\end{tabular}

Dados expressos em números absolutos e porcentagens (\%) ou média \pm desvio padrão (DP) ou mediana (P25-75);

*I.G.: idade gestacional; ${ }^{+}$P.N.: peso de nascimento.

Tabela 5. Amamentação na população estudada.

\begin{tabular}{|c|c|c|c|}
\hline \multicolumn{4}{|c|}{ Amamentação } \\
\hline & Sim & Não & $p$ \\
\hline Tipo de Parto & & & $<0,01$ \\
\hline Vaginal & $549(68,9)$ & $248(31,1)$ & \\
\hline Cesárea & $211(23,0)$ & $707(77,0)$ & \\
\hline Gemelaridade & & & $<0,01$ \\
\hline Sim & $6(7,1)$ & $79(92,9)$ & \\
\hline Não & $753(43,2)$ & $876(53,8)$ & \\
\hline I.G.* (semanas) & $39,0 \pm 1,4$ & $38,1 \pm 1,9$ & $<0,01$ \\
\hline P.N. ${ }^{+}$(gramas) & $3.224 \pm 455$ & $3.057 \pm 592$ & $<0,01$ \\
\hline Apgar $1^{\circ}$ minuto & $9(8-9)$ & $8(8-9)$ & $<0,01$ \\
\hline Apgar $5^{\circ}$ minuto & $9(9-9)$ & $9(9-9)$ & $<0,01$ \\
\hline Reanimação & & & $<0,01$ \\
\hline Sim & $4(9,5)$ & $38(90,5)$ & \\
\hline Não & $738(45,2)$ & $895(54,8)$ & \\
\hline Não Informado & $18(45,0)$ & $22(55,0)$ & \\
\hline Uso De Oxigenio & & & $<0,01$ \\
\hline Sim & $9(7,1)$ & $117(92,9)$ & \\
\hline Não & $711(48,0)$ & $769(52,0)$ & \\
\hline Não informado & $40(36,7)$ & $68(63,3)$ & \\
\hline
\end{tabular}

Dados expressos em números absolutos e porcentagens (\%) ou média \pm desvio padrão (DP) ou mediana (P25-75);

*I.G.: idade gestacional; ${ }^{+}$P.N.: peso de nascimento.

\section{DISCUSSÃO}

O serviço realizou o monitoramento dos indicadores de boas práticas em elevado percentual dos nascimentos, o que permitiu uma análise da realidade da aplicação dos mesmos nos pacientes elegíveis.

Houve elevada proporção de excluídos entre os nascidos vivos $(21,8 \%)$. Provavelmente isso ocorre devido às características da maternidade, que é terciária, referência no estado para partos prematuros, gemelares e de risco, assim como o predomínio de neonatos com comprometimento da vitalidade tanto por malformações maiores como secundário a patologias maternas na gestação. Observou-se também que muitos pacientes apresentaram mais de um motivo para exclusão, sendo justificado e marcado na ficha de acompanhamento aquele mais determinante no momento da assistência. O sexo masculino foi discretamente predominante em relação ao sexo feminino.

A via de parto mais frequente foi cesárea $(56,1 \%)$, proporção justificada devido à complexidade dos casos admitidos na 
unidade, onde muitas vezes é mandatória a resolução da gestação e compatível com os dados do SINASC de dezembro de 2018 onde a proporção de cesáreas foi de $55,99 \%{ }^{18} \mathrm{~A}$ frequência de partos gemelares $(4,6 \%)$ foi maior que a média nacional $(1,4 \%){ }^{2}$ Como o serviço tem um ambulatório de pré-natal para acompanhamento de gestações gemelares, o achado corresponde ao que se esperava.

Ao se avaliar isoladamente a aplicabilidade das medidas, o indicador com maior adesão foi o clampeamento oportuno do cordão, realizado em mais de dois terços da população elegível $(79,4 \%)$. A pior adesão foi à amamentação. Uma possível causa para o resultado seria o fato de, à época, se considerava ao preenchimento da ficha apenas a sucção nutritiva.

O clampeamento do cordão umbilical foi oportuno em mais de dois terços dos casos $(79,4 \%)$ e, ao compará-lo segundo a via de parto, foi determinante para um tempo de clampeamento oportuno nascer de parto vaginal (88,1\%) $(p<0,01)$. Justifica-se que os partos vaginais teriam um menor risco e os partos cesáreas seriam realizados em pacientes potencialmente mais graves. Em 13 partos, o clampeamento foi superior a três minutos. No entanto, atualmente, não há evidências da superioridade de benefícios em um tempo de clampeamento superior a três minutos. Quando se avaliou a presença de gestação gemelar, a maioria (60,7\%) teve o clampeamento em menos de um minuto de vida, em contraponto aos partos não gemelares, onde $81,5 \%$ conseguiram fazer o clampeamento oportuno. Não foram encontrados trabalhos na literatura para comparação. Nos casos em que houve necessidade de alguma manobra de reanimação, a maioria (86\%) teve o clampeamento precoce, em menos de um minuto após o nascimento. $\mathrm{O}$ dado é esperado, uma vez que, se não há início de movimento respiratório regular ou se o tônus muscular é flácido, as manobras iniciais de reanimação devem ser iniciadas e executadas em, no máximo, 30 segundos. ${ }^{19} \mathrm{Na}$ população que necessitou de oxigênio em algum momento, o grupo de clampeamento oportuno foi discretamente superior àqueles que realizaram clampeamento precoce. Quando avaliadas as variáveis independentes de peso e idade gestacional, a comparação entre os grupos que clampearam precoce e os que clampearam em mais de três minutos, bem como quando se compararam os grupos de clampeamento oportuno com os que clampearam em mais de três minutos, não foram estatisticamente significantes.

Houve diferença estatisticamente significante quanto ao Apgar ( $1^{\circ}$ e $5^{\circ}$ minutos) nos grupos de acordo com o tempo de clampeamento do cordão $(p<0.01)$. Tal dado se justifica uma vez que pacientes com maior gravidade têm clampeamento imediato do cordão. Na realização do Test Post-Roc (comparações múltiplas), a diferença foi significativa apenas entre os grupos que clampearam em menos de um minuto e entre um e três minutos, sem diferença estatisticamente significante quando comparados os grupos com clampeamentos menor que um minuto e maior que três minutos, bem como os entre um e três minutos e maior que três minutos $(p=0,92$ e $p=0,784)$.
No presente estudo a realização de contato pele a pele ocorreu em $66,7 \%$ dos pacientes. A adesão foi superior ao estudo realizado em outra grande maternidade do nordeste, ${ }^{20}$ onde avaliaram-se 107 mães e apenas dez realizaram contato pele a pele $(9,3 \%)$. Os resultados encontrados também foram superiores aos encontrados em estudos realizados no México $(9,0 \%)$ e Estados Unidos (17,3\%). ${ }^{21}$ Dado demonstra a adesão às práticas pela equipe.

Nesta pesquisa a via de parto foi determinante para a aplicação do contato pele a pele. A chance de realizar contato pele a pele é inferior ao se nascer de parto cesárea, sendo estatisticamente significativa $(p<0,01)$. É importante ressaltar que independente da via de parto, vaginal ou cesárea, o grupo que realizou o indicador foi superior ao grupo que não realizou o contato precoce. A presença de gemelaridade também foi determinante para a aplicabilidade desse indicador $(p<0,01)$. Não encontramos dados que tenham avaliado contato pele a pele em partos gemelares (Tabela 4).

Apesar da necessidade de alguma manobra de reanimação, 11 $(25,6 \%)$ bebês evoluíram bem e se beneficiaram do contato pele a pele na primeira hora de vida. Em todos os casos, a manobra realizada foi ventilação com pressão positiva. Houve necessidade de uso de oxigênio por períodos muito curtos em $39(30,5 \%)$ casos, com o RN evoluindo com boa saturação, sem desconforto respiratório e possibilidade de as boas práticas na sala de parto.

A introdução precoce da amamentação, bem como sua manutenção exclusiva nos seis primeiros meses pode prevenir cerca de $20 \%$ dos óbitos neonatais. O mesmo estudo estima que recém-nascidos que não foram amamentados na primeira hora de vida têm risco de morte aumentado em quatro vezes, quando comparados aos que foram amamentados. ${ }^{22}$ Neste trabalho, a via de parto também foi estatisticamente significante quanto a oportunidade ou não de se amamentar o bebê na primeira hora de vida. A maioria das mães que tiveram seus filhos por via vaginal amamentaram seus filhos (68,9\%), enquanto menos de um quarto dos bebês nascidos de cesárea foram amamentados na primeira hora de vida (23,0\%). A variável gemelaridade foi determinante para não amamentação na primeira hora de vida, onde se observou que a grande maioria dos conceptos $(92,9 \%)$ não foi amamentado na primeira hora de vida $(p<0,01)$. Uma pequena proporção daqueles reanimados $(9,5 \%)$ e dos que utilizaram inicialmente oxigênio $(7,1 \%)$ foram amamentados, também observadas como variáveis determinantes para a não amamentação na primeira hora de vida $(p<0,01)$ (Tabela 5).

Esta pesquisa verificou que $61,9 \%$ dos pacientes elegíveis realizaram os três indicadores de boas práticas. Dado superior ao encontrado por Lansky et al, ${ }^{1}$ em estudo multicêntrico realizado no Brasil, onde $0,9 \%$ dos neonatos foram beneficiados com boas práticas ao nascer.

Foi observado ainda que, houve justificativa para a não realização dos indicadores em 613 pacientes. A principal justificativa foi comprometimento da vitalidade do recémnascido, onde se explicou o não contato pele a pele e demais indicadores em 34,5\% dos conceptos. A partir dos 
dados obtidos, não encontramos critérios para exclusão dos indicadores. No entanto, há sinais e sintomas não descritos e que também impedem o contato pele a pele, como cianose ou hipotermia. Em 20,6\% dos casos a explicação para não se realizar os indicadores foi prematuridade. No entanto, nenhum deles com idade gestacional inferior a 34 semanas de idade gestacional. Condição materna foi a justificativa em $21,1 \%$ dos casos, os quais não tinham registro de admissão materna em UTI. Como o questionário não diferencia condição clínica de recusa materna, não foi possível identificar quantas mães não quiseram fazer e quantas não puderam fazer o contato pele a pele. Esta é uma limitação do estudo. Alguns pacientes não realizaram contato pele a pele ou estímulo a amamentação por limitações do serviço, onde em aproximadamente 11,3\% dos casos a explicação dada foi a ausência de profissional disponível (Gráfico 1).

Gráfico 1. Motivos de não execucão dos indicadores de boas práticas em pacientes elegíveis.

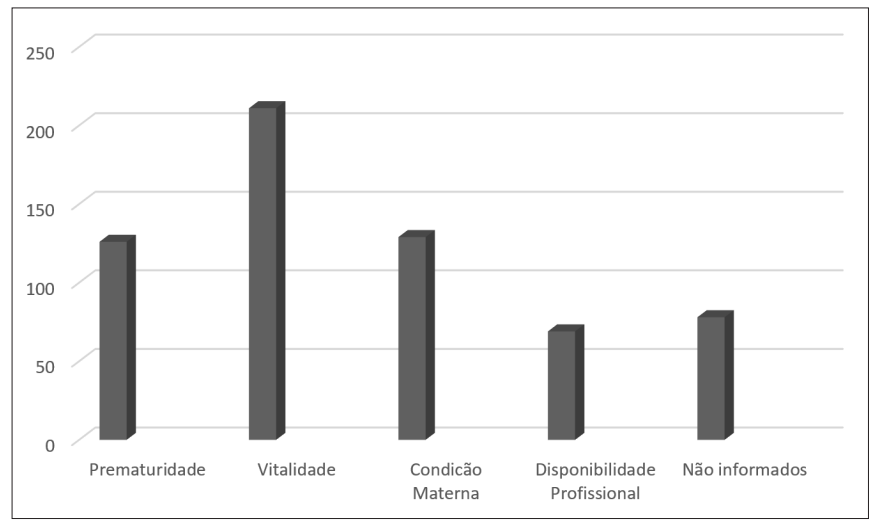

A avaliação destes resultados possibilita traçar estratégias para uma melhor adesão aos indicadores de boas práticas na assistência ao parto e recém-nascido, como: oferecer uma

\section{REFERÊNCIAS}

1. Lansky S, Friche AA, Silva AA, Campos D, Bittencourt SD, Carvalho ML, et al. Pesquisa Nascer no Brasil: perfil da mortalidade neonatal e avaliação da assistência à gestante e ao recém-nascido. Cad Saude Pública. 2014;30 Suppl 1:S1-15.

2 Brasil. Ministério da Saúde. DATASUS - Departamento de Informações e Análise Epidemiológica. Painel de Monitoramento de Nascidos Vivos [Internet]. Brasília: Ministério da Saúde; 2018 [acesso em: 30 jan 2019]. Disponível em: svs.aids.gov.br/dantps/ centrais-de-conteudos/paineis-de-monitoramento/natalidade/ nascidos-vivos

3. França E, Lansky S. Mortalidade infantil neonatal no Brasil: situação, tendências e perspectivas. Texto elaborado por solicitação da RIPSA para o Informe de Situação e Tendências: Demografia e Saúde, 2008 (Textos de Apoio, Texto 3) [acesso em: 20 dez 2018]. Disponível em: http://www.abep.org.br/publicacoes/index.php/anais/ article/view/1763

4. Liu L, Johnson HL, Cousens S, Perin J, Scott S, Lawn JE, et al. boa assistência pré-natal e, durante esse período, sensibilizar a mãe e esclarecer todos os benefícios da aplicabilidade dos indicadores; sensibilizar toda a equipe de assistência acerca das reais contraindicações, bem como a gestão possibilitar uma equipe em número e treinamento adequados.

\section{CONCLUSÃO}

As boas práticas na assistência ao parto são aplicadas na maioria dos pacientes nascidos no serviço. $\mathrm{O}$ clampeamento oportuno foi a mais aplicada.

O contato pele a pele e estímulo a amamentação na primeira hora de vida, além de melhorar a estabilidade térmica do $\mathrm{RN}$, com menos episódios de hipotermia, aumentam o vínculo da mãe com o seu filho e redução dos episódios de hipoglicemias neonatais. O fortalecimento deste vínculo facilita o estabelecimento do aleitamento materno exclusivo e a sua manutenção por mais tempo, com os seus benefícios já amplamente conhecidos.

O clampeamento oportuno do cordão umbilical, é prática recomendada em todos os partos pela Organização Mundial da Saúde desde 2007. Vários estudos confirmam os benefícios do clampeamento oportuno do cordão umbilical, como melhor estabilidade hemodinâmica e prevenção da anemia na infância, esta frequente até os dois anos de vida devido os níveis de ferro ao nascimento.

As boas práticas assistenciais durante o parto podem fazer muita diferença nas chances de sobrevivência e na saúde dos neonatos. Cabe aos profissionais de saúde a informação sobre os benefícios e se apropriar dessas práticas, demandando mudança de atitude da equipe e da rotina hospitalar. É necessária também a disseminação das informações à população, sendo uma estratégia fundamental para melhoria da qualidade de vida da criança, prognóstico neonatal e mortalidade.
Global, regional, and national causes of child mortality: an updated systematic analysis for 2010 with time trends since 2000. Lancet. 2012;379(9832):2151-61.

5. Moreira ME, Gama SG, Pereira AP, Silva AA, Lansky S, Pinheiro RS, et al. Práticas de atenção hospitalar ao recém-nascido saudável no Brasil. Cad de Saúde Pública. 2014;30 Sup:S128-S139.

6. Organizacao Mundial de Saúde. Recomendações da OMS para a prevenção e tratamento da hemorragia pós-parto [Internet]. Genebra: OMS; 2014 [acesso em: 20 dez 2018]. Disponível em: http://apps. who.int/iris/bitstream/handle/10665/75411/9789248548505_por. pdf? sequence $=12$.

7. Moore ER, Bergman N, Anderson GC, Medley N. Early skin-toskin contact for mothers and their healthy newborn infants. Cochrane Database Syst Rev. 2016;11:CD003519.

8. Oliveira FC, Assis KF, Martins MC, Prado MR, Ribeiro AQ, Sant'Ana LF, et al. Tempo de clampeamento e fatores associados 
à reserva de ferro de neonatos a termo. Rev Saúde Pública. 2014;48(1):10-18.

9. Silva CM, Pereira SC, Passos IR, Santos LC. Fatores associados ao contato pele a pele entre mãe/filho e amamentação na sala de parto. Rev Nutr. 2016;29(4):457-71.

10. Winberg J. Mother and newborn baby: mutual regulation of physiology and behavior- a selective review. Dev Psychobiology. 2005;47:217-29.

11. Edmond KM, Zandoh C, Quigley MA, Amenga-Etego S, OwusuAgyei S, Kirkwood BR. Delayed breastfeeding initiation increases risk of neonatal mortality. Pediatrics. 2006;117(3):e380-6.

12.Edmond KM, Kirkwood BR, Amenga-Etego S, Owusu-Agyei S, Hurt LS. Effect of early infant feeding practices on infection specific neonatal mortality: an investigation of the causal links with observational data from rural Ghana. Am J Clin Nutr. 2007;86:112631 .

13. Albesharat R, Ehrmann MA, Korakli M, Yazaji S, Vogel RF. Phenotypic and genotypic analyses of lactic acid bacteria in local fermented food, breast milk and faces of mothers and their babies. Syst Appl Microbiol. 2011;34:148-55.

14. Parm U, Metsvaht T, Sepp E, Ilmoja ML, Pisarev H, Pauskar $\mathrm{M}$, et al. Risk factors associated with gut and nasopharyngeal colonization by common Gram-negative species and yeasts in neonatal intensive care units patients. Early Hum Dev. 2011;87:391-9.

15.Castellote C, Casillas R, Ramírez-Santana C, Pérez-Cano FJ, Castell M, Moretones MG, et al. Premature delivery influences the immunological composition of colostrums and transitional and mature human milk. J Nutr. 2011;141:1181-7.
16. Araújo ED, Carbonare SB, Araújo MC, Palmeira P, Amaral JA, Sales VS. Total and specific IgA in colostrum and milk of mothers of Natal -Rio Grande do Norte, Brasil. Acta Cir Bras. 2005;20:178-84.

17. Brasil. Ministério da Saúde. Portaria $n^{\circ} 371$, de 7 de maio de 2014. Brasília: Ministério da Saúde; 2008 [acesso em: 7 fev 2007]. Disponível em: http://bvsms.saude.gov.br/bvs/saudelegis/sas/2014/ prt0371_07_05_2014.html

18. Brasil. Ministério da Saúde. DATASUS - Departamento de Informações e Análise Epidemiológica. Painel de Monitoramento de Nascidos Vivos segundo Classificação de Risco Epidemiológico (Grupos de Robson) [Internet]. Brasília: Ministério da Saúde; 2018 [acesso em: 30 jan 2019]. Disponível em: http://svs.aids.gov.br/ dantps/centrais-de-conteudos/paineis-de-monitoramento/natalidade/ grupos-de-robson/

19. Brasil. Sociedade Brasileira de Pediatria. Reanimação do recém-nascido $\geq 34$ semanas em sala de parto: diretrizes 2016 da Sociedade Brasileira de Pediatria de 26 de janeiro de 2016 [Internet]. Rio de Janeiro: SPB; 2016 [acesso em: 20 dez 2018]. Disponível em: http://www.sbp.com.br/fileadmin/user_upload/ DiretrizesSBPReanimacaoRNMaior34semanas26jan2016.pdf

20. Sampaio AR, Bousquat A, Barros C. Contato pele a pele ao nascer: um desafio a promoção do aleitamento materno em maternidade pública no Nordeste brasileiro com o título de Hospital Amigo da Criança. Epidemiol Serv Saúde. 2016;25(2):281-90.

21. Abdulghani N, Edvardsson K, Amir LH. Worldwide prevalence of mother-infant skin-to-skin contact after vaginal birth: a systematic review. PLoS One. 2018;13(10):e0205696.

22. Phukan D, Ranjan M, Dwivedi LK. Impact of timing of breastfeeding initiation on neonatal mortality in India. Int Breastfeed J. 2018;13:27.

\section{Como citar:}

Patrício ML, Paes LS, Castro EC, Barreto GA, Patrício MP. Indicadores de boas práticas na assistência neonatal ao parto em maternidade terciária em Fortaleza. Rev Med UFC. 2021;61(1):1-9. 